\title{
Producer's Best Response in Pay-as-clear Electricity Market with Uncertain Demand
}

\author{
D. Aussel ${ }^{1}$, M. Branda ${ }^{2}$, R. Henrion ${ }^{3}$, M. Pištěk ${ }^{2}$ \\ ${ }^{1}$ Lab. PROMES UPR CNRS 8521, University of Perpignan, \\ Perpignan, France \\ aussel@univ-perp.fr \\ ${ }^{2}$ The Czech Academy of Sciences, UTIA \\ Pod Vodárenskou věží 4, 18208 Prague 8, Czech Republic \\ branda@utia.cas.cz; pistek@utia.cas.cz \\ ${ }^{3}$ Weierstrass Institute for Applied Analysis and Stochastics \\ Mohrenstr. 39, 10117 Berlin, Germany \\ henrion@wias-berlin.de
}

\section{Extended Abstract}

The non-cooperative characteristic of electricity markets led to concentrate on Nash equilibria and multi-leader-follower games, where the producers of electricity are viewed as leaders while the regulator of the market, referred as the Independent System Operator (ISO), is viewed as the common follower. The Nash equilibrium associated with this problem is the equilibrium state in which the market should operate ideally. Due to different evolutions - the influence of renewable energy, the introduction of smart-grid, or fusion of European markets - models of the electricity markets need to be constantly updated and/or adapted, see, e.g. [2,3,4].

In this work, we are particularly focused on several sources of uncertainty in (pay-as-clear) electricity markets. To deal with stochastic demand we employ the so-called chance constrained formulations of the problem [1] of the ISO as well as the problem of each producer. In detail, the ISO minimizes the production cost using a value-at-risk (VaR) approach, thus hedging against discrepancies between estimated and real electricity demand. Similarly, in the day-ahead market, each producer is hedging against the uncertainty of his own prediction of the demand using VaR approach again. In such a setting we aim at determining the "best response" of a given producer, i.e. the bid maximizing its profit.

To solve such a bi-level problem, one has to start with the chance-constrained problem of the ISO. Neglecting transportation thermal losses, the chance constraint has a structure of the so-called separable (random) right-hand side, and so it can be reformulated using the quantile function (i.e. the inverse of the distribution function). Transforming thus the chance constraint into a deterministic constraint, the solution of the ISO problem may be found explicitly following [2,3]. In the second step, we substitute this solution into the formula determining the profit of a producer. Benefiting from the specific structure of this formula, we may reformulate the problem of a producer as a deterministic nonlinear programming equivalent. The resulting problem is then solved numerically to find the best response of a given producer.

To illustrate our results, we provide a numerical evaluation based on the historical distribution of both estimated and real electricity demand. We used the real market data from France (source: www.rte-france.com) observed between January 3 and February 28 of 2017. Based on these observations, we estimated the parameters of the respective lognormal distributions. Note that the mean values correspond to the predicted quantities, whereas the variances represent the mean squared prediction errors. Based on these estimates we determined the optimal production of electricity given the probability prescribed to satisfy the aggregated demand. Then, we prepared a numerical simulation with five producers and found the best response of one of them using the sequential quadratic programming algorithm. Finally, we investigated the development of the best response of a given producer with respect to the changes of the probabilistic level.

This research has been supported by grants GA17-08182S and GA18-04145S of the Czech Science Foundation.

\section{References}

[1] L. Adam and M. Branda, "Nonlinear chance constrained problems: Optimality conditions, regularization and solvers," Journal of Optimization Theory and Applications, vol. 170, no. 2, pp. 419-436, 2016. 
[2] D. Aussel and P. Bendotti and M. Pištěk, "Nash equilibrium in a pay-as-bid electricity market: Part 1 - existence and characterization," Optimization, vol. 66, no. 6, pp. 1013-1025, 2017.

[3] D. Aussel and P. Bendotti and M. Pištěk, "Nash equilibrium in a pay-as-bid electricity market part 2 - best response of a producer," Optimization, vol. 66, no. 6, pp. 1027-1053, 2017.

[4] R. Henrion and J.V. Outrata and T. Surowiec, "Analysis of M-stationary points to an EPEC modeling oligopolistic competition in an electricity spot market," ESAIM: Control, Optim. and Calculus of Variations, vol. 18, pp. 296-317, 2012. 